\title{
Analysis of mechanical and electrical damages in piezoelectric ceramics
}

\author{
Xinhua Yang, Guowei Zeng and Weizhong Cao \\ Huazhong University of Science and Technology, \\ China
}

\section{Introduction}

For recent decades, due to the intrinsic electromechanical coupling, piezoelectric ceramics have been used widely in the modern aeronautical and astronautic engineering, the medical apparatus and the instruments, etc. However, their brittleness might result in damage and failure. Therefore, it is very important theoretically and practically for fracture analysis, reliability design and lifetime prediction of piezoelectric ceramics to investigate the fracture mechanism.

On the basis of linear piezoelectric fracture mechanics, some solutions were obtained by Pak (1990, 1992), Sosa and Pak (1990), Sosa (1991, 1992), Suo et al (1992), Suo (1993) and others, but they contradict the piezoelectric fracture experiments (Park and Sun, 1995; Heyer et al, 1998). The linear piezoelectric fracture mechanics predicted that the stress intensity factors were independent of the applied electric field, but the experimental results (Park and Sun, 1995; Heyer et al, 1998) showed that an existing electric field might impede or accelerate crack propagation, which was related to the applied electric field direction.

Regarding piezoelectric ceramics as a class of mechanically brittle and electrically ductile solids, Gao et al (1997) put forward a strip saturation model analogous with the classical Dugdale model for plastic yielding, in which electrical displacement is assumed to yield when electric field is up to its linear limit. Yang and Zhu (1998) thought that piezoelectric nonlinearity comes from domain switching, so they proposed a small-scale domain-switching model to explain the toughening mechanism. Fulton and Gao (2001) proposed an electrical nonlinearity model based on piezoelectric microstructure. They simulated polarization switching and saturation in ferroelectrics by using a collection of discrete electric dipoles superimposed on a medium satisfying the linear piezoelectric constitutive law, and then derived a local crack driving force which generates a qualitative match to experimental observations. Based on the field limiting space charge model, Zhang et al (2003) proposed a charge-free zone model, and estimated the electric field at the tip of an electrically conductive crack according to the electric field intensity factor. Assuming that the electric field in a strip ahead of the crack tip is equal to the dielectric breakdown strength, Zhang et al (2005) developed a strip dielectric breakdown model similar to the strip saturation model. 
In the above nonlinear models, only electrical nonlinearity is considered. However, the experiments (Zhang and Gao, 2004) demonstrated that in addition to the nonlinearities in the relationships of polarization versus electric field and strain versus electric field, the stress-strain curves are nonlinear. From the viewpoint of continuum damage theory, material nonlinearity is caused by damage, so that the continuum damage mechanics can be extended to describe nonlinear behaviors of piezoelectric ceramics. Mizuno (2002) introduced a single damage variable into the constitutive equation of piezoelectric ceramics to take the effect of damage on material properties into account, and after that, Mizuno and Honda (2005) analyzed the steady-state crack growth in a double cantilever piezoelectric beam.

In our work, an anisotropic damage constitutive model involving mechanical and electrical damages was proposed (Yang et al, 2003 and 2005a), and the phenomenological damage tensors were qualitatively connected with microstructure parameters by the unit cell method (Yang et al, 2007 and 2008; Zeng et al, 2008). The linear and nonlinear piezoelectric fracture criteria were established after the damage analysis of the well-known piezoelectric fracture experiments of Park and Sun's (Yang et al, 2005b). Finally, the damage constitutive model and the nonlinear fracture criterion were used to investigate the quasi-static propagation behavior of the midspan crack in a three-point bending PZT-4 beam, and effects of mechanical and electric loads on crack growth were observed.

\section{Piezoelectric damage constitutive model}

Damage in a piezoelectric solid can come from a number of sources, including fabrication, connection, service, etc. After homogenization, a damaged material element from the solid is regarded as a homogeneous continuum, so that its constitutive behavior can be characterized by new three-dimensional anisotropic constitutive equations with $\tilde{\mathbf{c}}, \tilde{\mathbf{e}}$, and $\tilde{\lambda}$ as the elastic, piezoelectric and dielectric constants.

$$
\sigma_{i j}=\tilde{c}_{i j k l} \varepsilon_{k l}-\tilde{e}_{k i j} E_{k}, \quad D_{i}=\tilde{e}_{i k l} \varepsilon_{k l}+\tilde{\lambda}_{i k} E_{k} .
$$

These constants are called effective elastic, piezoelectric, and dielectric constants, respectively. They are related to the undamaged constants $\mathbf{c}, \mathbf{e}$, and $\boldsymbol{\lambda}$ by

$$
\begin{aligned}
& \tilde{c}_{i j m n}=\frac{1}{4}\left(\omega_{k i} \delta_{l j}+\delta_{k i} \omega_{l j}\right)\left(\omega_{o m} \delta_{p n}+\delta_{o m} \omega_{p n}\right) c_{k l o p}, \\
& \tilde{e}_{i m n}=\frac{1}{2}\left(\omega_{k m} \delta_{l n}+\delta_{k m} \omega_{l n}\right) G_{j i} e_{j k l}, \quad \tilde{\lambda}_{i l}=G_{j i} G_{k l} \lambda_{j k} .
\end{aligned}
$$

where $\boldsymbol{\omega}$ and $\mathbf{G}$ are two second-order continuity tensors, and $\boldsymbol{\delta}$ is the Kronecker Delta. The mechanical and electrical damage variables $\mathrm{D}^{\mathrm{M}}$ and $\mathrm{D}^{\mathrm{E}}$, which characterize damage degree of the material element, can be expressed in terms of the continuity tensors as

$$
D_{i j}^{\mathrm{M}}=\delta_{i j}-\omega_{i k} \omega_{k j}, \quad D_{i j}^{\mathrm{E}}=\delta_{i j}-G_{i k} G_{k j} .
$$

Eq.(1) combined with Eqs.(2) and (3) are called piezoelectric damage constitutive model. It can be rewritten in matrix form as follows. 


$$
\{\sigma\}=[\tilde{c}]\{\varepsilon\}-[\tilde{e}]^{\mathrm{T}}\{E\}, \quad\{D\}=[\tilde{e}]\{\varepsilon\}+[\tilde{\lambda}]\{E\} .
$$

where

$$
\begin{aligned}
& \{\sigma\}=\left[\begin{array}{llllll}
\sigma_{11} & \sigma_{22} & \sigma_{33} & \tau_{23} & \tau_{13} & \tau_{12}
\end{array}\right]^{\mathrm{T}}, \\
& \{\varepsilon\}=\left[\begin{array}{llllll}
\varepsilon_{11} & \varepsilon_{22} & \varepsilon_{33} & \gamma_{23} & \gamma_{13} & \gamma_{12}
\end{array}\right]^{\mathrm{T}} \text {, } \\
& \{E\}=\left[\begin{array}{lll}
E_{1} & E_{2} & E_{3}
\end{array}\right]^{\mathrm{T}}, \quad\{D\}=\left[\begin{array}{lll}
D_{1} & D_{2} & D_{3}
\end{array}\right]^{\mathrm{T}} \text {. }
\end{aligned}
$$

And according to Eq.(2), we have

$$
[\tilde{c}]=[N]^{\mathrm{T}}[c][N], \quad[\tilde{e}]=[G]^{\mathrm{T}} e[N], \quad[\tilde{\lambda}]=[G]^{\mathrm{T}}[\lambda][G] .
$$

where

$$
\begin{aligned}
{[N] } & =\left[\begin{array}{cccccc}
\omega_{11} & 0 & 0 & 0 & \frac{1}{2} \omega_{13} & \frac{1}{2} \omega_{12} \\
0 & \omega_{22} & 0 & \frac{1}{2} \omega_{23} & 0 & \frac{1}{2} \omega_{12} \\
0 & 0 & \omega_{33} & \frac{1}{2} \omega_{23} & \frac{1}{2} \omega_{13} & 0 \\
0 & \omega_{23} & \omega_{23} & \frac{1}{2}\left(\omega_{22}+\omega_{33}\right) & \frac{1}{2} \omega_{12} & \frac{1}{2} \omega_{13} \\
\omega_{13} & 0 & \omega_{13} & \frac{1}{2} \omega_{12} & \frac{1}{2}\left(\omega_{11}+\omega_{33}\right) & \frac{1}{2} \omega_{23} \\
\omega_{12} & \omega_{12} & 0 & \frac{1}{2} \omega_{13} & \frac{1}{2} \omega_{23} & \frac{1}{2}\left(\omega_{11}+\omega_{22}\right)
\end{array}\right], \\
{[G] } & =\left[\begin{array}{lll}
G_{11} & G_{12} & G_{13} \\
G_{12} & G_{22} & G_{23} \\
G_{13} & G_{23} & G_{33}
\end{array}\right] .
\end{aligned}
$$

The transversely isotropic constant matrices can be expressed as follows

$$
\begin{aligned}
{[c] } & =\left[\begin{array}{cccccc}
c_{11} & c_{12} & c_{13} & 0 & 0 & 0 \\
c_{12} & c_{11} & c_{13} & 0 & 0 & 0 \\
c_{13} & c_{13} & c_{33} & 0 & 0 & 0 \\
0 & 0 & 0 & c_{44} & 0 & 0 \\
0 & 0 & 0 & 0 & c_{44} & 0 \\
0 & 0 & 0 & 0 & 0 & \frac{1}{2}\left(c_{11}-c_{12}\right)
\end{array}\right], \\
{[e] } & =\left[\begin{array}{cccccc}
0 & 0 & 0 & 0 & e_{15} & 0 \\
0 & 0 & 0 & e_{15} & 0 & 0 \\
e_{31} & e_{31} & e_{33} & 0 & 0 & 0
\end{array}\right],[\lambda]=\left[\begin{array}{ccc}
\lambda_{11} & 0 & 0 \\
0 & \lambda_{11} & 0 \\
0 & 0 & \lambda_{33}
\end{array}\right] .
\end{aligned}
$$

If damage is transversely isotropic and has the same principal axes as the material, Eq.(7) can be reduced to 


$$
\begin{aligned}
{[N] } & =\left[\begin{array}{cccccc}
\omega_{11} & 0 & 0 & 0 & 0 & 0 \\
0 & \omega_{11} & 0 & 0 & 0 & 0 \\
0 & 0 & \omega_{33} & 0 & 0 & 0 \\
0 & 0 & 0 & \frac{1}{2}\left(\omega_{11}+\omega_{33}\right) & 0 & 0 \\
0 & 0 & 0 & 0 & \frac{1}{2}\left(\omega_{11}+\omega_{33}\right) & 0 \\
0 & 0 & 0 & 0 & 0 & \omega_{11}
\end{array}\right], \\
{[G] } & =\left[\begin{array}{ccc}
G_{11} & 0 & 0 \\
0 & G_{11} & 0 \\
0 & 0 & G_{33}
\end{array}\right] .
\end{aligned}
$$

Accordingly, only four scalar quantities are needed for transversely isotropic piezoelectric damage, namely, $\omega_{11}, \omega_{33}, G_{11}$, and $G_{33}$, and the correponding four damage variables are

$$
\begin{aligned}
& D_{11}^{\mathrm{M}}=1-\omega_{11}^{2}=1-\frac{\tilde{c}_{11}}{c_{11}}, \quad D_{33}^{\mathrm{M}}=1-\omega_{33}^{2}=1-\frac{\tilde{c}_{33}}{c_{33}}, \\
& D_{11}^{\mathrm{E}}=1-G_{11}^{2}=1-\frac{\tilde{\lambda}_{11}}{\lambda_{11}}, \quad D_{33}^{\mathrm{E}}=1-G_{33}^{2}=1-\frac{\tilde{\lambda}_{33}}{\lambda_{33}} .
\end{aligned}
$$

\section{Relation between damages and microstructure parameters}

It is well known that damage degree depends on microstructure geometry, density, interactions, etc. As the first step, it needs to be solved how the phenomenological damage tensors is quantitatively connected with microstructure parameters. Eq.(10) reveals a way to determine quantitative connections between the mechanical and electrical damages and microstructure parameters, because the effective properties of the damaged material element can be calculated by means of micromechanical mechanics methods, such as the unit cell method.

\subsection{Insulating microvoids}

PZT-7A piezoelectric ceramics with the material constants listed in Table 1 is assumed to contain many periodically distributed ellipsoidal insulating microvoids, shown in Fig.1(a). As the smallest periodical unit which contains sufficient microstructure information, a unit cell is taken out from the material, and only quarter of the cell model needs to be considered because of symmetry. See Fig.1(b). Accordingly, the displacement boundary conditions of the model can be described as

$$
\begin{array}{lll}
u_{1}=0 & \text { for } & x_{1}=0 \\
u_{2}=0 & \text { for } & x_{2}=0 \\
u_{2}=0 & \text { for } & x_{3}=0
\end{array}
$$




\begin{tabular}{|c|c|c|c|c|c|c|c|c|c|c|}
\hline Materials & $c_{11}$ & $c_{12}$ & $c_{13}$ & $c_{33}$ & $c_{44}$ & $e_{31}$ & $e_{33}$ & $e_{15}$ & $\lambda_{11}$ & $\lambda_{33}$ \\
\hline & \multicolumn{5}{|c|}{$\mathrm{GPa}$} & \multicolumn{3}{|c|}{$\mathrm{C} / \mathrm{m}^{2}$} & \multicolumn{2}{|c|}{$10-9 \mathrm{~F} / \mathrm{m}$} \\
\hline PZT-7A & 148 & 76.2 & 74.2 & 131 & 25.4 & -2.1 & 9.5 & 9.2 & 4.07 & 2.08 \\
\hline PZT-4 & 139 & 77.8 & 74.3 & 113 & 25.6 & -6.98 & 13.84 & 13.44 & 6 & 5.47 \\
\hline
\end{tabular}

Table 1. Material properties

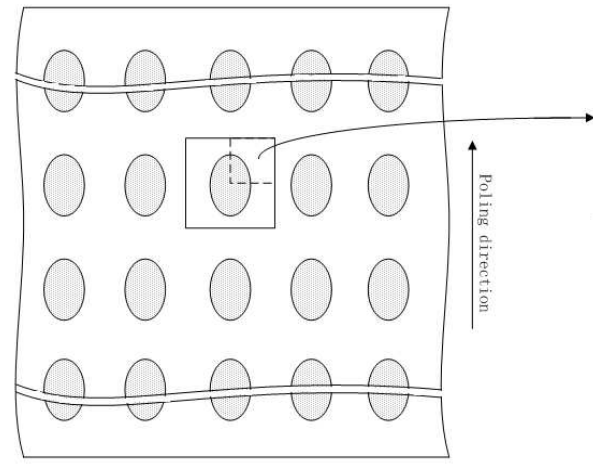

(a) PZT-7A with periodically distributed microvoids Fig. 1. Computational model

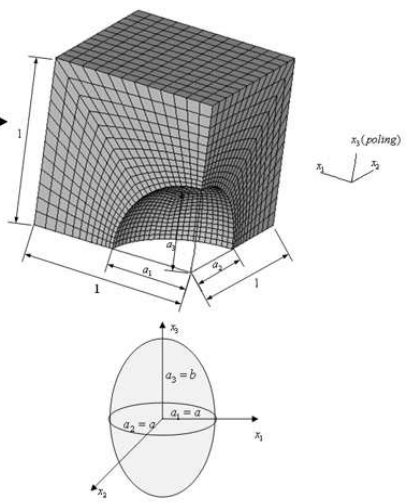

(b) Quarter of the cell model

Different loading and boundary conditions are imposed on the cell model in order to evaluate its effective properties. For example, when the displacement degree of freedom $U_{x_{1}=1}=1$ is applied in the boundary planes $x_{1}=1$ or $U_{x_{3}=1}=1$ applied in the planes $x_{3}=1$, the effective elastic constant $\tilde{c}_{11}$ can be evaluated from Eq. (4) by

$$
\begin{aligned}
& E_{1}=E_{2}=E_{3}=0, \quad \varepsilon_{22}=\varepsilon_{33}=0, \quad \varepsilon_{11} \neq 0, \\
& \tilde{c}_{11}=\frac{\sigma_{11}}{\varepsilon_{11}} \approx \frac{Q_{x_{1}=1}}{U_{x_{1}=1}} .
\end{aligned}
$$

And $\tilde{c}_{33}$ can also be evaluated by

$$
\begin{aligned}
& E_{1}=E_{2}=E_{3}=0, \quad \varepsilon_{11}=\varepsilon_{22}=0, \quad \varepsilon_{33} \neq 0, \\
& \tilde{c}_{33}=\frac{\sigma_{33}}{\varepsilon_{33}} \approx \frac{Q_{x_{3}=1}}{U_{x_{3}=1}} .
\end{aligned}
$$

In the above two expressions, $Q_{x_{1}=1}$ and $Q_{x_{3}=1}$ are the active force in the planes $x_{1}=1$ and $x_{3}=1$, respectively. When only the electric field $E_{2}$ is imposed along the $x_{2}$-direction through applying the voltage $V_{x_{2}=1}$ in the plane $x_{2}=1$ and $V_{x_{2}=0}$ in the plane $x_{2}=0$ of the cell, we have $D_{2}=\tilde{e}_{15} \varepsilon_{32}+\tilde{\lambda}_{33} E_{2}$, so that the dielectric constant $\tilde{\lambda}_{11}$ can be evaluated by 


$$
\begin{aligned}
& E_{1}=E_{3}=0, E_{2} \neq 0, \quad \varepsilon_{11}=\varepsilon_{22}=\varepsilon_{33}=\varepsilon_{32}=0, \\
& \tilde{\lambda}_{11}=\frac{D_{2}}{E_{2}} \approx \frac{D_{x_{2}=1}}{V_{x_{2}=1}-V_{x_{2}=0}}
\end{aligned}
$$

Applying the voltage $V_{x_{3}=1}$ in the plane $x_{3}=1$ and $V_{x_{3}=0}$ in the plane $x_{3}=0$ of the cell, the dielectric constant $\tilde{\lambda}_{33}$ can be obtained by

$$
\begin{aligned}
& E_{1}=E_{2}=0, E_{3} \neq 0, \quad \varepsilon_{11}=\varepsilon_{22}=\varepsilon_{33}=0, \\
& \tilde{\lambda}_{33}=\frac{D_{3}}{E_{3}} \approx \frac{D_{x_{3}=1}}{V_{x_{3}=1}-V_{x_{3}=0}}
\end{aligned}
$$

In the above two expressions, $D_{x_{2}=1}$ and $D_{x_{3}=1}$ are the average components of electric flux density in the planes $x_{2}=1$ and $x_{3}=1$, respectively. Further, the mechanical and electrical damages can be determined in accordance with Eq.(10).

Similarly, the piezoelectric coefficients can be evaluated by

and

$$
\begin{aligned}
& E_{3} \neq 0, \quad \varepsilon_{11}=\varepsilon_{22}=\varepsilon_{33}=0, \\
& \tilde{e}_{13}=-\frac{\sigma_{11}}{E_{3}} \approx-\frac{F_{x_{1}=1}}{V_{x_{3}=1}-V_{x_{3}=0}}, \quad \tilde{e}_{33}=-\frac{\sigma_{11}}{E_{3}} \approx-\frac{F_{x_{3}=1}}{V_{x_{3}=1}-V_{x_{3}=0}}
\end{aligned}
$$

$$
\begin{aligned}
& E_{2} \neq 0, \quad \varepsilon_{11}=\varepsilon_{22}=\varepsilon_{33}=\varepsilon_{32}=0, \\
& \tilde{e}_{15}=-\frac{\sigma_{32}}{E_{2}} \approx \frac{F_{x_{2}=1}}{V_{x_{2}=1}-V_{x_{2}=0}}
\end{aligned}
$$

In the above two expressions, $F_{x_{1}=1}, F_{x_{2}=1}$ and $F_{x_{3}=1}$ are the reactive forces on the planes $x_{1}=1, x_{2}=1$ and $x_{3}=1$, respectively. On the other hand, according to the obtained mechanical and electrical damage components, the piezoelectric coefficients can be also obtained from Eq.(6). By comparison of these results from the above two kinds of method, it can be checked if the hypothesis of transversely isotropic damage is rational.

In the unit cell, the ellipsoidal void geometry is characterized by its volume fraction $f=V_{\mathrm{v}} / V_{\mathrm{m}}$, where $V_{\mathrm{v}}$ and $V_{\mathrm{m}}$ are respectively the void and matrix volumes, and aspect ratio $S=b / a$, where $a$ and $b$ are respectively the minor and major semi-axes of the void. The longitudinal cross-section area fraction of ellipsoidal void can be expressed by $A_{3}=(3 f \sqrt{\pi} / 4 S)^{\frac{2}{3}}$ and the transverse cross-section area fraction by $A_{1}=A_{2}=(3 f \sqrt{\pi S} / 4)^{\frac{2}{3}}$.

The void aspect ratio $S$ is fixed at 1.0 and the void volume fraction $f$ is changed from 0 to $32.1 \%$, in order to evaluate the influences of the void volume fraction $f$ on the damages. The variation curves of damages with the void volume fraction $f$ are drawn in Fig.2(a). It is apparent that both the mechanical and electrical damages increase with the increasing void volume fraction $f$ and the curves of $D_{11}^{\mathrm{M}}$ and $D_{33}^{\mathrm{M}}$ superpose each other, which reveals that the mechanical damage induced by spherical voids is isotropic. This is in good agreement 
with our knowledge. And then, the void volume fraction $f$ is fixed at $6.54 \%$ and the void aspect ratio $S$ is changed from 0.3 to 2.0, in order to observe influences of the void aspect ratio $S$ on the damages. The variation curves of the damages with the void aspect ratio $S$ are plotted in Fig.2 (b). While $D_{33}^{\mathrm{M}}$ goes down rapidly in forepart but slowly afterward with the increasing void aspect ratio $S, D_{11}^{\mathrm{M}}$ always goes up slowly. This is understandable because $A_{3}$ decreases but $A_{1}$ or $A_{2}$ increases with the increasing aspect ratio $S$ for a fixed volume fraction $f$. Nevertheless, $D_{11}^{\mathrm{E}}$ and $D_{33}^{\mathrm{E}}$ hardly change, which shows that the electrical damage components are independent of the void aspect ratio $S$.

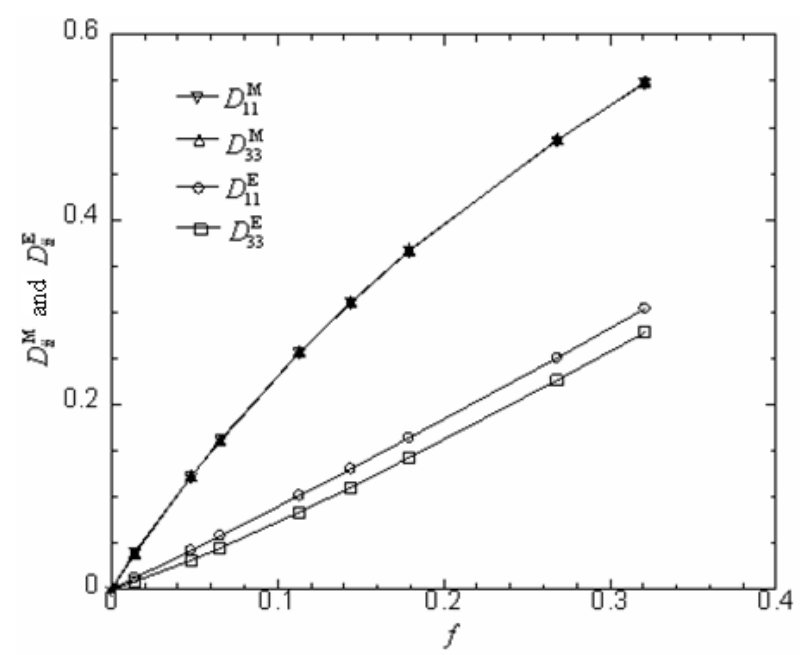

(a)

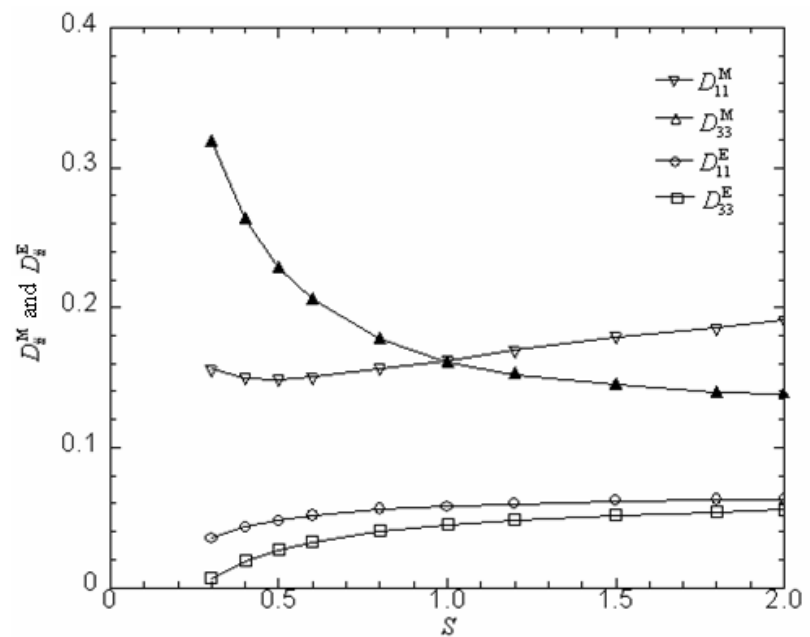

(b)

Fig. 2. Variation curves of damages with (a) volume fraction and (b) aspect ratio of an insulating void 
The effective piezoelectric coefficients are calculated from Eq.(6) and from Eq.(16) or (17), respectively, and the variation curves of the effective piezoelectric coefficients with the void volume fraction and the void aspect ratio are drawn in Fig.3 (a) and (b), respectively. Apparently, the results from the two methods are very approximate, which reflects that the hypothesis of transversely isotropic damage is reasonable for the analyzed piezoelectric solid with voids.

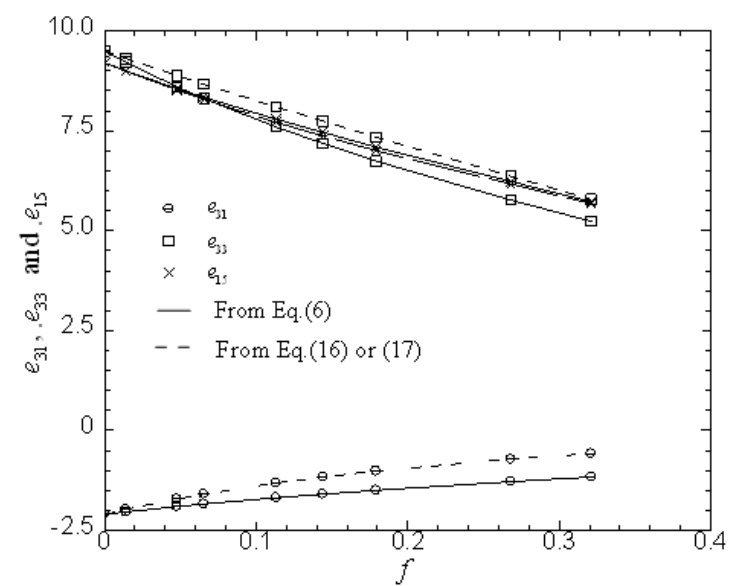

(a)

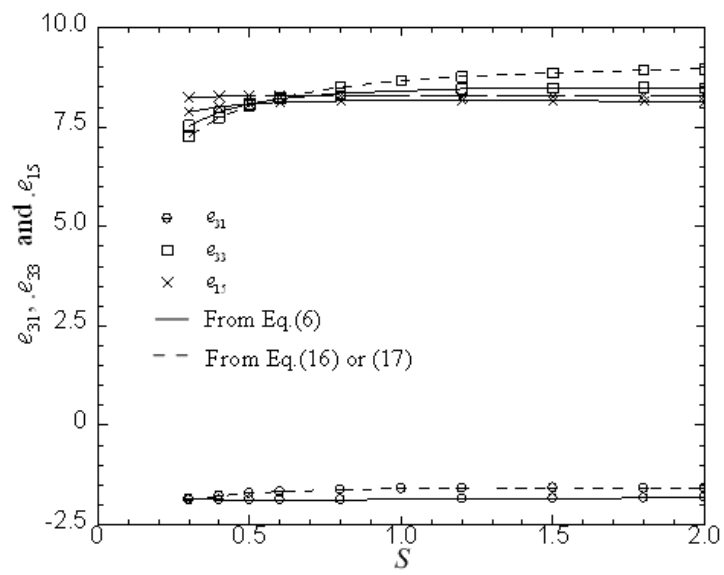

(b)

Fig. 3. Variation curves of effective piezoelectric coefficients with (a) the void volume fraction and (b) the void aspect ratio

\subsection{Conductive microvoids}

For conductive microvoids, the shape ratio $S$ is fixed at 1.0 (namely the microvoids are spherical) and the volume fraction $f$ is changed from 0 to $32.1 \%$, in order to investigate the effect of the 
volume fraction on damages. The variation curves of mechanical and electrical damages with the volume fraction are plotted in Fig.4(a). It can be found that both the mechanical and electrical damage components increase with the increasing volume fraction and the curves of $D_{11}^{\mathrm{M}}$ and $D_{33}^{\mathrm{M}}$ overlap each other. After that, the volume fraction $f$ is fixed at $6.54 \%$ and the shape ratio $S$ is changed from 0.4 to 2 in order to investigate the effect of the shape ratio on damages. The variation curves of mechanical and electrical damages with the shape ratio are plotted in Fig.4(b). For the mechanical damage components, $D_{33}^{\mathrm{M}}$ rapidly decreases but $D_{11}^{\mathrm{M}}$ slowly increases with the increasing shape ratio. For the electrical damage components, however, $D_{11}^{\mathrm{E}}$ slowly increases but $D_{33}^{\mathrm{E}}$ slowly decreases with the increasing shape ratio.

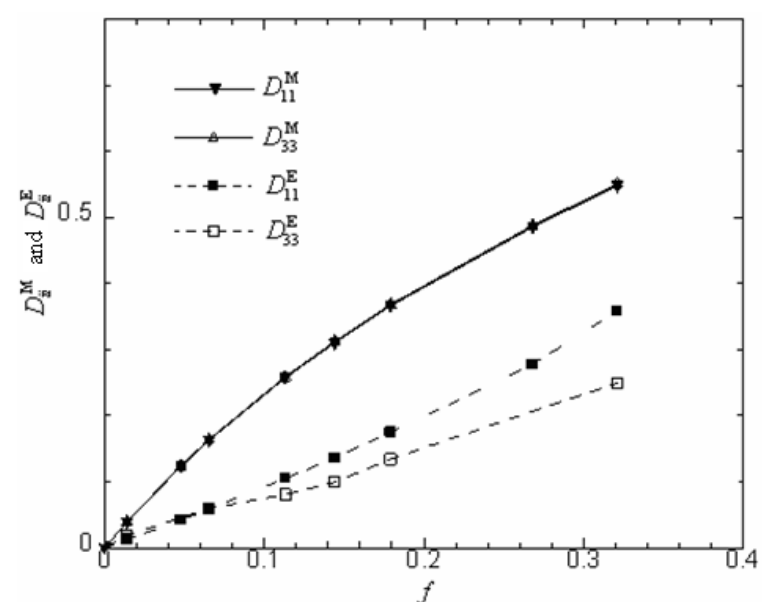

(a)

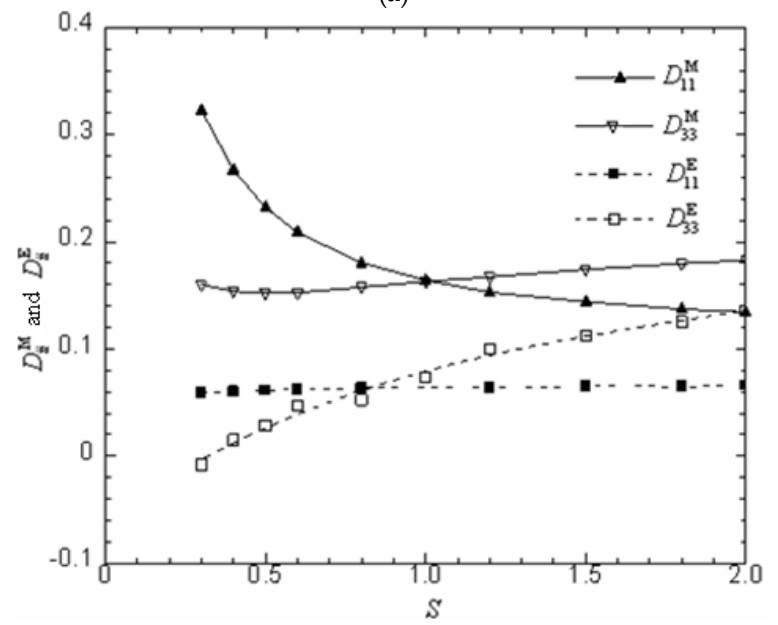

(b)

Fig. 4. Variation curves of damages with (a) the volume fraction and (b) the aspect ratio for conducting voids 


\subsection{Conductive inclusions}

Consider a PZT-7A piezoelectric solid with periodically distributed ellipsoidal conductive inclusions, shown in Fig.5. The solid is polarized in the $z$ direction. The inclusions have Poisson's ratio of 0.25 and elastic modulus of $E=\frac{5}{6} \alpha c_{11}$. In order to evaluate the effect of inconlusion elastic modulus on damages, we fix $f$ in $6.54 \%$ and $S$ in 1 , and change $\alpha$ from 0 to 1 . The curves of mechanical and electrical damages with $\alpha$ are plotted in Fig.6. It can be seen that the mechanical damage rapidly decreases as $\alpha$ increases, and goes to zero when $\alpha$ tends toward 1. This is because inclusions transform into microvoids when $\alpha=0$ and approximates to the matrix material when $\alpha=1$. However, the electric damage slowly increases when $\alpha$ increases, because the variation of $\alpha$ does not change the conductivity of inclusions. Obviously, the inclusion elastic modulus has great influence on the mechanical damage but a little on the electric damage.

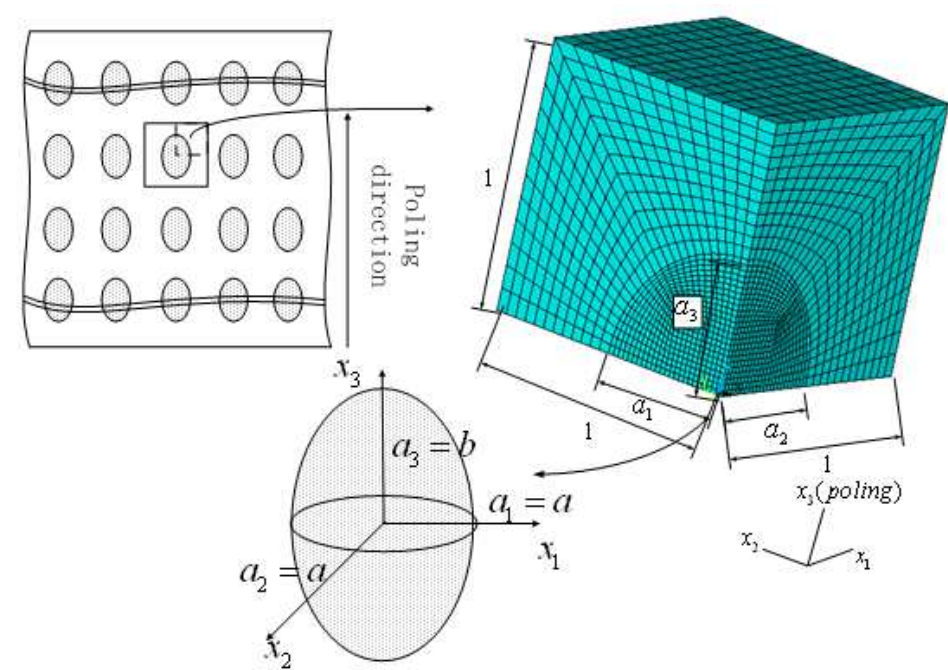

(a)

(b)

Fig. 5. Unit cell model and its coordinate system: (a) PZT-7A with periodically distributed inclusions; (b) 1/8 model of the cell 


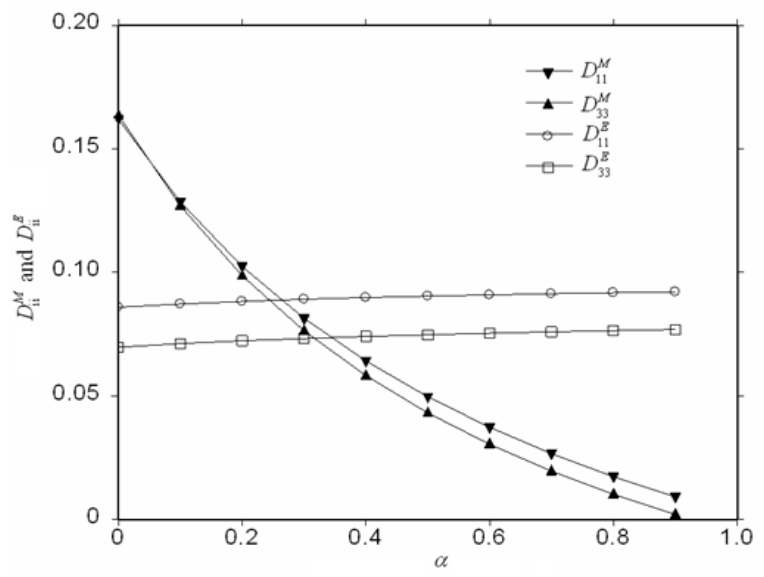

Fig. 6. Variation curves of mechanical and electrical damages with elastic coefficient ratio

In succession, we fix $S$ in 1 and set $\alpha$ as 0.4 and 0.6 , respectively, to investigate variation law of mechanical and electrical damages with inclusion volume fraction. The curves are drawn in Fig.7. It can be found that mechanical and electrical damages monotonously increase with inclusion volume fraction for different elastic moduli. This is possibly because increasing inclusion volume has greater influence on electromechanical properties of the materials.

Finally, the effects of inclusion shape on damages are discussed, when $f$ is fixed in $6.54 \%$ and $S$ is set as 0.4 and 0.6 , respectively. The computational results are shown in Fig.8. It is easily found that inclusion shape has very complex influence on mechanical and electrical damages, but the variation curves of damages with inclusion shape ratio have similar shape for different elastic moduli. Moreover, the inclusion shape variation has a little influence on the mechanical damage, but great influence on the electrical damage. For a constant inclusion volume, when the shape ratio increases, $A_{3}$ decreases, but $A_{1}$ and $A_{2}$ increase, so that $D_{11}^{\mathrm{E}}$ and $D_{33}^{\mathrm{E}}$ have opposite variation laws.

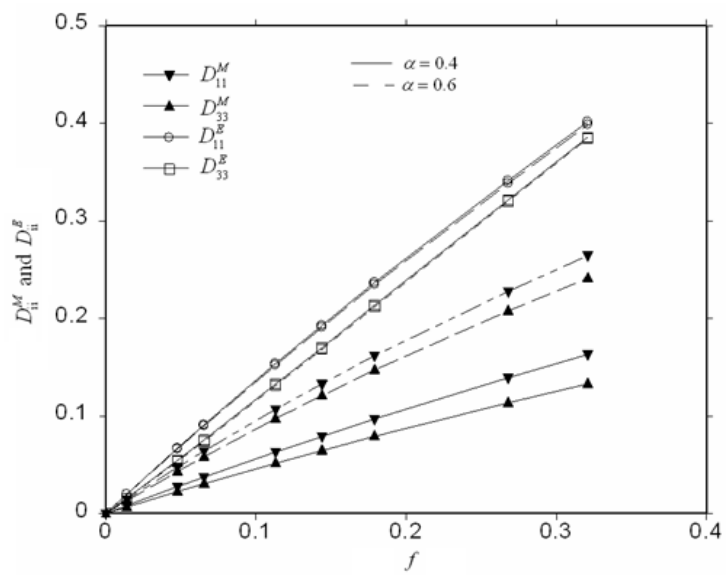

Fig. 7. Variation curves of mechanical and electrical damages with the volume fraction 


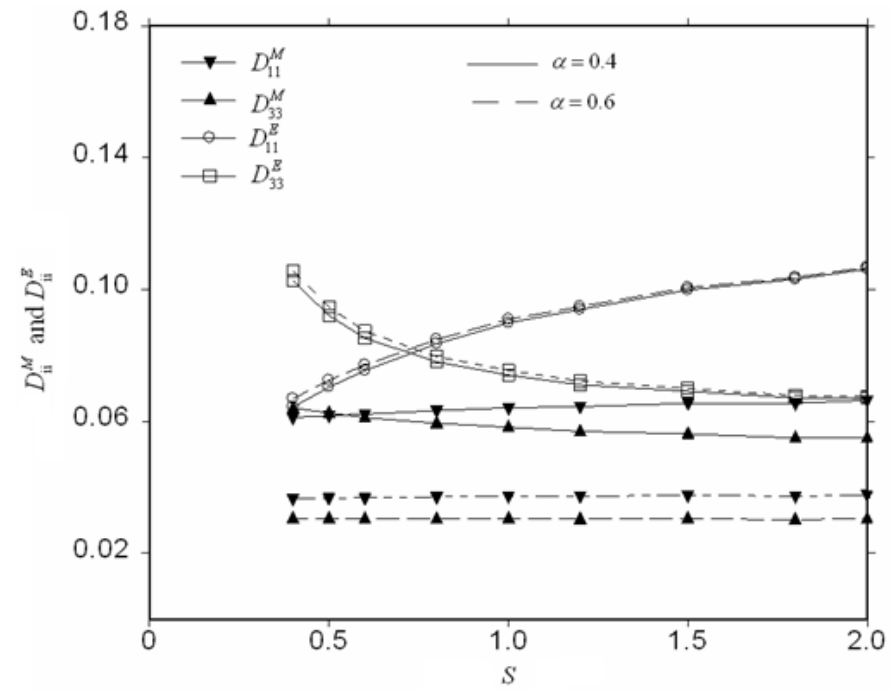

Fig. 8. Variation curves of mechanical and electrical damages with the aspect ratio

\section{Fracture criteria}

For the brittle piezoelectric ceramics, the onset of crack growth almost inevitably results in failure, so it is very important for understanding piezoelectric fracture behaviors to establish a useful fracture criterion. While the natural extension of linear elastic fracture mechanics provided an agreeably simple and general criterion (Suo et al, 1992), it was not supported by experimental studies (Park and Sun, 1995; Heyer et al, 1998). Arguing that fracture was a mechanical process and should be controlled only by the mechanical part of the energy, Park and Sun (1995) used the maximum mechanical strain energy release rate for predicting fracture loads. However, there was no fundamental reason to separate a physical process into two parts: mechanical and electrical. Shen and Nishioka (2000), Sih and Zuo (2000), and Zuo and Sih (2000) proposed an energy density criterion.

In order to establish a damage-based fracture criterion, the damage constitutive equations in Section 2 are used to characterize the constitutive behavior of PZT-4 piezoelectric ceramics. The undamaged properties are given in Table 1 . The specimens are respectively subjected to different critical mechanical loads and their corresponding electric fields given by Park and Sun (1995). The mechanical and electrical damages near the crack tips in the compact tension specimens and the three-point bending specimens are computed.

The damage components $D_{33}^{\mathrm{M}}$ and $D_{33}^{\mathrm{E}}$ are dominant in the problems under consideration, so only these two components at crack-tips are observed. Their relationship is imitated by means of linear and nonlinear methods, and the parameters in the equations are calculated according to the method of least squares.

$$
\begin{aligned}
& D_{33}^{\mathrm{M}}=0.5238+0.5651 D_{33}^{\mathrm{E}}, \\
& D_{33}^{\mathrm{M}}=0.5029+0.8878 D_{33}^{\mathrm{E}}+3.5384\left(D_{33}^{\mathrm{E}}\right)^{2} .
\end{aligned}
$$


The curves are plotted in Fig.9. It is apparent that the nonlinear imitation is better than the linear. Eq.(18) can be rewritten as

$$
\begin{aligned}
& 1.9092 D_{33}^{\mathrm{M}}-1.0789 D_{33}^{\mathrm{E}}=1, \\
& 1.9885 D_{33}^{\mathrm{M}}-1.7653 D_{33}^{\mathrm{E}}-7.0361\left(D_{33}^{\mathrm{E}}\right)^{2}=1 .
\end{aligned}
$$

Define (1) linearly combined damage:

$$
D=a_{1} D_{33}^{\mathrm{M}}+a_{2} D_{33}^{\mathrm{E}}
$$

and (2) nonlinearly combined damage:

$$
D=b_{0} D_{33}^{\mathrm{M}}+b_{1} D_{33}^{\mathrm{E}}+b_{3}\left(D_{33}^{\mathrm{E}}\right)^{2} .
$$

In the above two formulations, coefficients $a_{1}, a_{2}, b_{0}, b_{1}$, and $b_{2}$ are material dependent, but independent of geometries, loading conditions and boundary conditions of specimens. For PZT-4, $a_{1}=1.9092, a_{2}=-1.0789 ; b_{0}=1.9885, b_{1}=-1.7653, b_{2}=-7.0361$. Accordingly, a piezoelectric fracture criterion can be given as follows.

$$
D=D_{\text {c }}
$$

where, $D_{\mathrm{c}}=1$. This means that piezoelectric fracture occurs when the combined damage $D$ reaches its critical value $D_{\mathrm{c}}$.

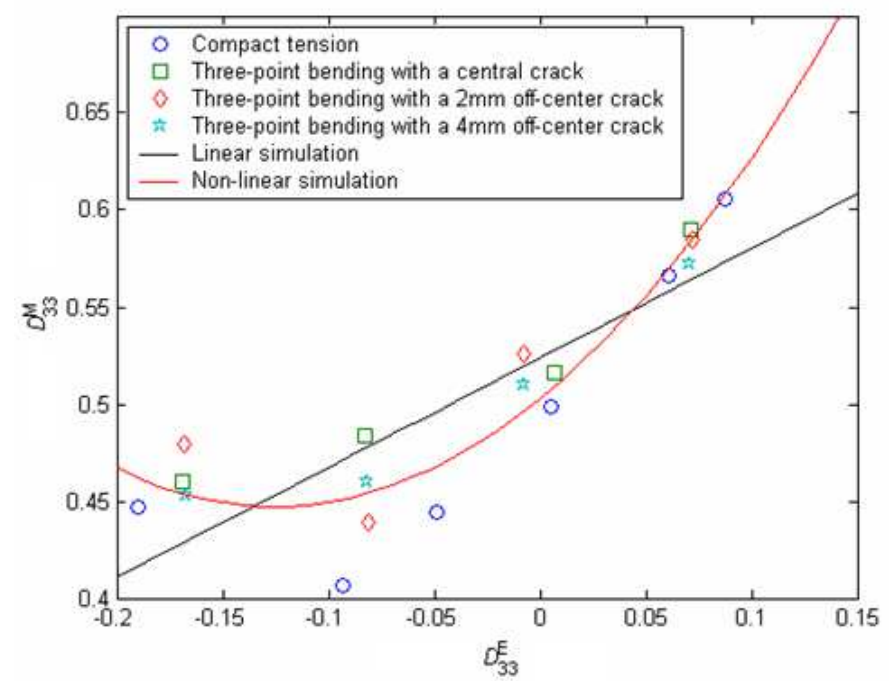

Fig. 9. Linear and nonlinear imitations of mechanical and electrical damages at the crack-tips 


\section{Quasi-static crack propagation in piezoelectric beam}

As an important application of the aformentioned piezoelectric damage constitutive model and nonlinear fracture criteria, the quasi-static propagation behaviors of the midspan crack in a three-point bending PZT-4 beam with sizes of $19.1 \mathrm{~mm} \times 9 \mathrm{~mm} \times 5.1 \mathrm{~mm}$ are simulated in different mechanically and electrically loading conditions. The beam is homogeneously poled along the $x_{3}$-direction and subjected to a downward concentrated force $F$ in the midspan and an electric voltage $U$ excitateding an electric field parallel to its length direction. A midspan insulating edge crack with a depth of $4 \mathrm{~mm}$ is perpendicular to the poling direction. The combined-damage determined by the dominant mechanical and electrical damage components is regarded as the fracture criterion and its gradient is assumed to control cracking direction. The influences of mechanical and electrical loads on the crack growth rate are evaluated.

\subsection{Effect of mechanical load on crack propagation}

Referring to the experimental data by Park and Sun (1995), we fix the electrical load at $U=3 \mathrm{KV}$ and change the mechanical load $F$ from $400 \mathrm{~N}$ to $500 \mathrm{~N}, 600 \mathrm{~N}, 700 \mathrm{~N}$ and $800 \mathrm{~N}$ in order to evaluate the effect of mechanical load on crack propagation. The crack growth increment $\Delta a$ at different computational steps is observed, and its variation and fitting curves with the computational step $T$ are drawn in Fig.10. It can be seen that the crack propagation has an increasingly accelerating trend and the mechanical load plays a very important role in crack growth. As an example, within the computational step range given in this paper, the crack depth increment $\Delta a$ has the minimum lower limit $(0.5 \pm 0.01) \times 10^{-4} \mathrm{~mm}$ at the start and the minimum upper limit $\quad(0.96 \pm 0.01) \times 10^{-4} \mathrm{~mm}$ at the final computational step for the external concentrated force $F=400 \mathrm{~N}$, but the maximum lower limits $(1.1 \pm 0.01) \times 10^{-4} \mathrm{~mm}$ and the maximum upper limit $(1.79 \pm 0.01) \times 10^{-4} \mathrm{~mm}$ for $F=800 \mathrm{~N}$. Accordingly, it is very obvious that mechanical load enhances crack propagation. Fig.11 exhibits the effect of mechanical load on the variation curve of the crack depth with the computational step.

Mechanical loading is a dominant factor controlling piezoelectric fracture. This is why the conventional piezoelectric fracture theories using the stress intensity factor (Pak, 1992; Sosa, 1992; Suo, 1992), the energy release rate (Park and Sun, 1995; Gao et al, 1997) and the energy density factor (Shen and Nishioka, 2000; Sih and Zuo, 2000; Zuo and Sih, 2000) as fracture criteria can give good fracture predictions for high stress cases. 


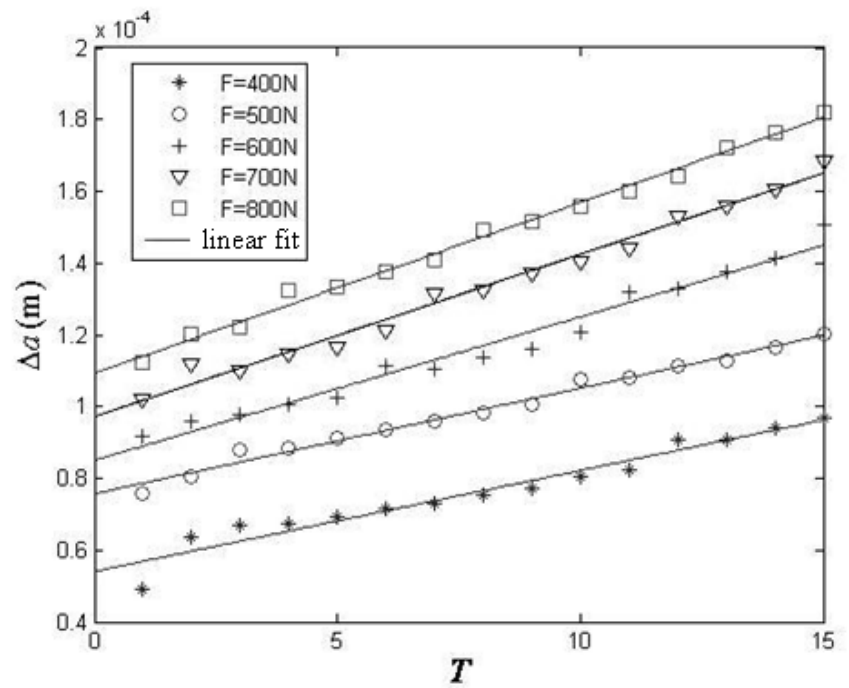

Fig. 10. Crack growth rate curves at different mechanical loads

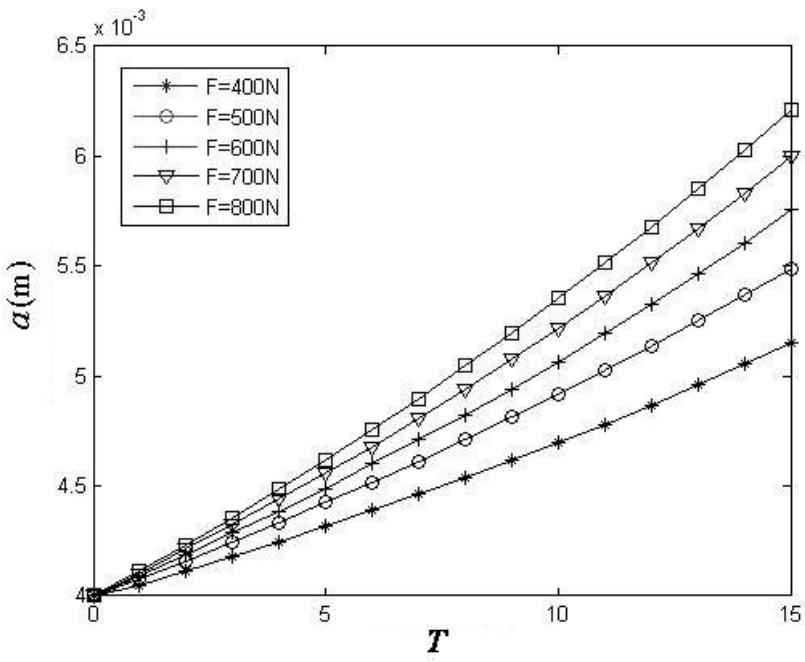

Fig. 11. Crack depth curves at different mechanical loads

\subsection{Effect of electric field on crack propagation}

Electric field is another important influencing factor. To observe the electromechanical coupling fracture behavior of piezoelectric media, Tobin and Pak (1993) early performed a series of indentation tests on PZT-8 ceramics under a given static electric field and an indentation load of $4.9 \mathrm{~N}$ on different locations. The results showed that the cracks perpendicular to the poling direction became longer or shorter under a positive or negative 
electric field. It was concluded that the positive field assisted the crack propagation, whereas the negative field retarded it. The analogous conclusion was also given by Park and Sun (1995) after completing the compact tension and three-point bending tests for PZT-4 piezoelectric ceramics.

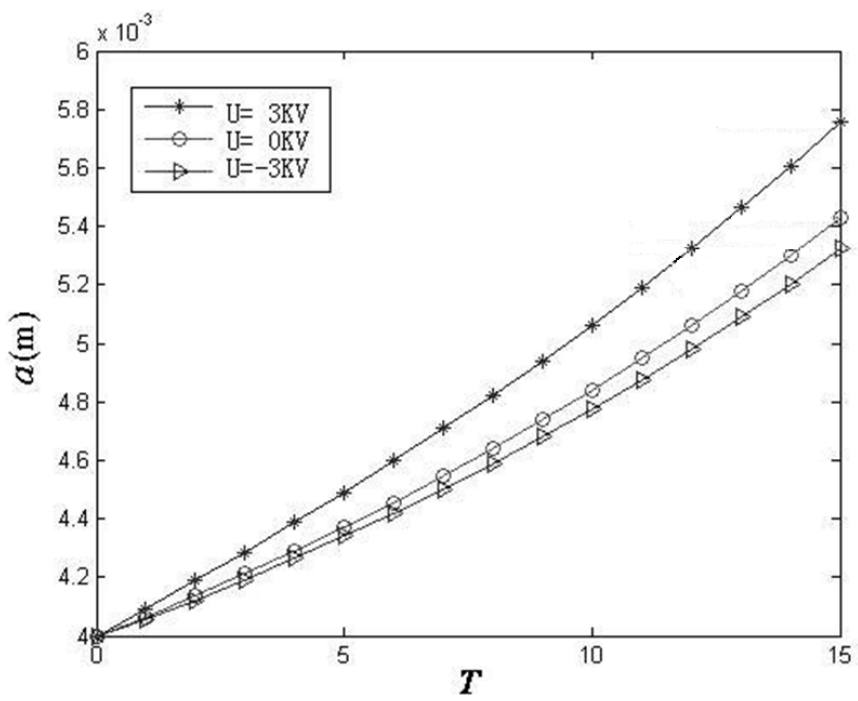

Fig. 12. Crack depth curves at different electrical loads

In order to evaluate the influence of electric field on crack propagation, an invariable mechanical load of $F=600 \mathrm{~N}$ is imposed on the beam, and the electrical voltage $U$ is changed from $3 \mathrm{KV}$ to $0 \mathrm{KV}$ and $-3 \mathrm{KV}$. Some results are plotted in Fig.12. It is apparent that the applied electric field has great effect on the crack propagation. The crack growth rate comes to its maximum for the electrical voltage of $3 \mathrm{KV}$. Along with the reducing voltage, however, the crack growth rate decreases rapidly, and reaches the minimum value at the electrical voltage of $-3 \mathrm{KV}$. It can be concluded that a positive electric field enhances crack propagation, whereas a negative electric field inhibits it. This is qualitatively consistent with the experimental conclusions given by Park and Sun (1995), and Tobin and Pak (1993).

\section{Conclusions}

In this chapter, a three-dimensional anisotropic piezoelectric damage constitutive model is developed by extension of continuum damage mechanics into piezoelectric materials. Using the unit cell method, the quantitative connections between the phenomenological damage tensors and microstructure parameters are established. These discussed microstructures include: (1) periodically distributed insulating microvoids, (2) conductive microvoids, and (3) conductive inclusions. Based on the compact tension tests and the three-point bending tests performed by Park and Sun, damage-based linear and nonlinear piezoelectric fracture criteria are obtained by using the method of least squares combined with numerical damage analysis for the fracture critical cases. As an application example, both the proposed 
piezoelectric damage constitutive model and the nonlinear fracture criterion are used to investigate the quasi-static propagation behavior of the midspan crack in a three-point bending PZT-4 beam, and the influences of mechanical and electrical loads on cracking behavior are evaluated.

\section{References}

Fulton, C.C. \& Gao, H. (2001). Effect of local polarization switching on piezoelectric fracture. Journal of the Mechanics and Physics of Solids, Vol. 49, pp. 927 - 952.

Gao, H.; Zhang, T.Y., \& Tong, P. (1997). Local and global energy release rates for an electrically yielded crack in a piezoelectric ceramic. J. Mech. Phys. Solids. Vol. 45, pp. 491-510.

Heyer, V.; Schneider, G.A.; Balke, H.; Drescher, J. \& Bahr, H.A. (1998). A fracture criterion for conducting cracks in homogeneously poled piezoelectric PZT-PIC 151 ceramics. Acta Mater. Vol. 46, pp. 6615-6622.

Mizuno, M. (2002). Constitutive equation of piezoelectric ceramics taking into account damage development. Key Eng. Mater. Vol. 233-236, pp. 89-94.

Mizuno, M. \& Honda, Y. (2005). Simplified analysis of steady-state crack growth of piezoelectric ceramics based on the continuum damage mechanics. Acta Mech. Vol. 179, pp. 157-168.

Pak, Y.E. (1990). Crack extension force in a piezoelectric material. Trans. ASME J. Appl. Mech. Vol. 57, pp. 647-653.

Pak, Y.E. (1992). Linear electro-elastic fracture mechanics of piezoelectric materials. Int. J. Fracture Vol. 54, pp. 79-100.

Park, S.B. \& Sun, C.T. (1995). Fracture criteria of piezoelectric ceramics. J. Am. Ceram. Soc. Vol. 78, pp. 1475-1480.

Shen, S. \& Nishioka, T. (2000). Fracture of piezoelectric materials: energy density criterion. Theor. Appl. Fract. Mech. Vol. 33, pp. 57-65.

Sih, G.C. \& Zuo, J.Z. (2000). Multiscale behavior of crack initiation and growth in piezoelectric ceramics. Theor. Appl. Fract. Mech. Vol. 34, pp. 123-141.

Sosa, H. (1991). Plane problems in piezoelectric media with defects. Int. J. Solids Struct. Vol. 28, pp. 491-505.

Sosa, H. (1992). On the fracture mechanics of piezoelectric solids. Int. J. Solids Struct. Vol. 29, pp. 2613-2622.

Sosa, H. \& Pak, Y.E. (1990). Three-dimensional eigenfunction analysis of a crack in a piezoelectric material. Int. J. Solids Struct. Vol. 26, pp. 1-15.

Suo, Z. (1993). Models for breakdown-resistant dielectric and ferroelectric ceramics. J. Mech. Phys. Solids. Vol. 41, pp. 1155-1176.

Suo, Z.; Kuo, C.M.; Barnett, D.M. \& Willis, J.R. (1992). Fracture mechanics for piezoelectric ceramics. J. Mech. Phys. Solids. Vol. 40, pp. 739-765.

Tobin, A.G. \& Pak, Y.E. (1993). Effect of electric fields on fracture behavior of PZT ceramics. Proc. SPIE--Int. Soc. Opt. Eng. Vol. 1916, pp. 78-86.

Yang, W. \& Zhu, T. (1998). Switch-toughening of ferroelectrics subjected to electric field. Journal of the Mechanics and Physics of Solids, Vol. 46, pp. 291-311.

Yang, X.H.; Chen, C.Y. \& Hu, Y.T. (2003). Analysis of damage near a conducting crack in a piezoelectric ceramic. Acta Mechanica Solida Sinica, Vol. 16, pp. 147-154. 
Yang, X.H.; Chen, C.Y.; Hu, Y.T. \& Wang, C. (2005). Damage analysis and fracture criteria for piezoelectric ceramics. International Journal of Non-Linear Mechanics, Vol. 40, pp. 1204-1213.

Yang, X.H.; Zeng, G.W. \& Chen, C.Y. (2007). Determination of Mechanical and Electrical Damages of Piezoelectric Material with Periodically Distributed Microvoids. Journal of Mechanics, Vol. 23, pp. 107-112.

Yang, X.H.; Zeng, G.W. \& Chen, C.Y. (2008). Analysis of Mechanical and Electrical Damages of Piezoelectric Ceramic with Periodically Distributed Conducting Inclusions. Journal of Mechanical Strength, Vol. 30, pp. 844-847. (In Chinese)

Yang, X.H.; Chen, C.Y.; Hu, Y.T. \& Wang, C. (2005). Combined damage fracture criteria for piezoelectric ceramics. Acta Mechanica Solida Sinica, Vol. 18, pp. 21-27.

Zeng, G.W.; Yang, X.H. \& Chen, C.Y. (2008). Mechanical and electrical damages of piezoelectric ceramics with periodically distributed conductive voids. Journal of Wuhan University of Science and Technology, Vol. 31, pp. 424-426. (In Chinese)

Zhang, T.Y.; Wang, T.H. \& Zhao, M.H. (2003). Failure behavior and failure criterion of conductive cracks (deep notches) in thermally depoled PZT-4 ceramics. Acta Materialia, Vol. 51, pp. 4881-4895

Zhang, T.Y.; Zhao, M.H. \& Gao, C.F. (2005). The strip dielectric breakdown model. International Journal of Fracture, Vol. 132, pp. 311-327.

Zhang, T.Y. \& Gao, C.F. (2004). Fracture behaviors of piezoelectric materials. Theoretical and Applied Fracture Mechanics, Vol. 41, pp. 339-379.

Zuo, J.Z. \& Sih, G.C. (2000). Energy density theory formulation and interpretation of cracking behavior for piezoelectric ceramics. Theor. Appl. Fract. Mech. Vol. 34, pp. 17-33. 


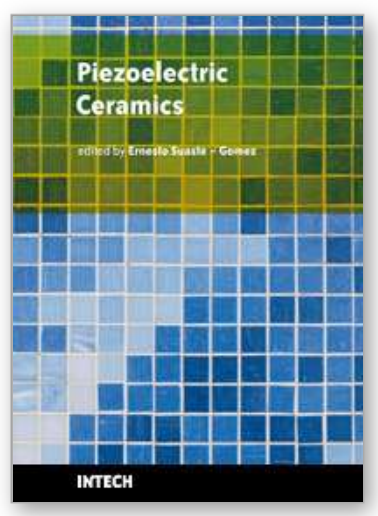

\author{
Piezoelectric Ceramics \\ Edited by Ernesto Suaste-Gomez
}

ISBN 978-953-307-122-0

Hard cover, 294 pages

Publisher Sciyo

Published online 05, October, 2010

Published in print edition October, 2010

This book reviews a big window of opportunity for piezoelectric ceramics, such as new materials, material combinations, structures, damages and porosity effects. In addition, applications of sensors, actuators, transducers for ultrasonic imaging, positioning systems, energy harvesting, biomedical and microelectronic devices are described. The book consists of fourteen chapters. The genetic algorithm is used for identification of RLC parameters in the equivalent electrical circuit of piezoelectric transducers. Concept and development perspectives for piezoelectric energy harvesting are described. The characterization of principal properties and advantages of a novel device called ceramic-controlled piezoelectric with a Pt wire implant is included. Biocompatibility studies between piezoelectric ceramic material and biological cell suspension are exposed. Thus, piezoelectric ceramics have been a very favorable solution as a consequence of its high energy density and the variety of fabrication techniques to obtain bulk or thin films devices. Finally, the readers will perceive a trend analysis and examine recent developments in different fields of applications of piezoelectric ceramics.

\title{
How to reference
}

In order to correctly reference this scholarly work, feel free to copy and paste the following:

Xinhua Yang, Guowei Zeng and Weizhong Cao (2010). Analysis of Mechanical and Electrical Damages in Piezoelectric Ceramics, Piezoelectric Ceramics, Ernesto Suaste-Gomez (Ed.), ISBN: 978-953-307-122-0, InTech, Available from: http://www.intechopen.com/books/piezoelectric-ceramics/analysis-of-mechanical-andelectrical-damages-in-piezoelectric-ceramics

\section{INTECH}

open science | open minds

\section{InTech Europe}

University Campus STeP Ri

Slavka Krautzeka 83/A

51000 Rijeka, Croatia

Phone: +385 (51) 770447

Fax: +385 (51) 686166

www.intechopen.com

\section{InTech China}

Unit 405, Office Block, Hotel Equatorial Shanghai

No.65, Yan An Road (West), Shanghai, 200040, China

中国上海市延安西路65号上海国际贵都大饭店办公楼 405 单元

Phone: +86-21-62489820

Fax: +86-21-62489821 
(C) 2010 The Author(s). Licensee IntechOpen. This chapter is distributed under the terms of the Creative Commons Attribution-NonCommercialShareAlike-3.0 License, which permits use, distribution and reproduction for non-commercial purposes, provided the original is properly cited and derivative works building on this content are distributed under the same license. 